\title{
An Implementation Plan for Integrated Control and Asset Management of Petroleum Production Facilities
}

\author{
Atalla F. Sayda and James H. Taylor
}

\begin{abstract}
This paper addresses innovative issues of asset management for the petroleum industry, which is very crucial for profitable oil and gas facilities operations and maintenance. A research project was initiated to study the feasibility of an intelligent asset management system. Having proposed a conceptual model for such a system in previous work [1], [2], we describe its behavior in terms of data and control flow, and pave the way for an implementation and rapid prototyping plan for such system. Furthermore we discuss the required off-the-shelf development tools. A simplified system prototype is introduced as a colored petri net model, which will be used to analyze the prototype logical structure and dynamic performance. We finally discuss the project progress status and future work.
\end{abstract}

\section{INTRODUCTION}

Asset management in modern process plants has become a very challenging process, especially in abnormal situations. This can be attributed to the size and complexity of modern process plants and increasingly massive information overload. The management and control of such plants involve many tasks of different time-scale and complexity including data reconciliation and fusion, fault detection, isolation, and accommodation (FDIA), process model identification and optimization, and supervisory control. The automation of these complementary tasks within an information and control infrastructure will reduce maintenance expenses, improve utilization and output of manufacturing equipment, enhance safety, and improve product quality. Many research studies proposed different combinations of systems theoretic and artificial intelligence techniques to tackle the asset management problem, and delineated the requirements of such system [3], [4], [5].

Several conceptual frameworks have been suggested for modeling complex intelligent systems in the past two decades, such as expert systems, whose implementation results revealed several drawbacks, namely, lack of learning mechanisms and weak representation power [6], [7]. Newell et al proposed another promising framework, cognitive architectures, which models human cognition and problem solving behavior [8], [9]. Multi-agent systems (MAS), which can be considered as an instantiation of distributed artificial intelligence, are another conceptual framework for modeling complex systems. A MAS is defined as a loosely coupled network of problem solvers that work together to

James H. Taylor is with the Department of Electrical \& Computer Engineering, University of New Brunswick, PO Box 4400, Fredericton, NB CANADA E3B 5A3 jtayloreunb. ca

Atalla F. Sayda is a PhD candidate with the Department of Electrical \& Computer Engineering, University of New Brunswick, PO Box 4400, Fredericton, NB CANADA E3B 5A3 atalla.sayda@unb. ca solve problems, that are beyond their individual capabilities [10], [11]. The MAS platform emphasizes distribution, autonomy, interaction (i.e., communication), coordination, and organization of individual agents.

Having defined the conceptual model of an automated asset management system and its architecture in previous work [1], [2], this paper paves the way for another project milestone, and proposes an implementation road map for such a system and different development tools to be used. The paper is organized as follows: First, we review asset management projects being developed around the world. Then we discuss our project and its objectives followed by a detailed behavioral model of the system in terms of data and control flow mechanisms. Then, we discuss the implementation plan and the required development tools. After that we describe a plan for a simple prototype which will be the cornerstone in our research project. Finally, we conclude with future research and development steps.

\section{WORLD-WIDE ASSET MANAGEMENT PROJECTS}

The first research program to address the ASM problem was the Pilots Associate (PA) program, which is a joint effort of the Defense Advanced Research Projects Agency and the US Air Force, managed by the Air Force's Wright Laboratory. The program began in February 1986 as an application demonstration for DARPA's Strategic Computing Initiative. A primary goal of the PA program was to enhance combat fighter pilot effectiveness by increasing pilots' situational awareness and decreasing their workload. DARPA wanted to advance the programs technology base, principally in the area of real-time, cooperating knowledgebased systems. The Air Force wanted to explore the potential of intelligent systems applications to improve the effectiveness and survivability of post-1995 fighter aircraft.

The Pilots Associate concept developed as a set of cooperating, knowledge-based subsystems: two assessor and two planning subsystems, and a pilot interface. The two assessors, Situation Assessment and System Status, determine the state of the outside world and the aircraft systems, respectively. The two planners, Tactics Planner and Mission Planner, react to the dynamic environment by responding to immediate threats and their effects on the prebriefed mission plan. The Pilot-Vehicle Interface subsystem provides the critical connection between the pilot and the rest of the system [12], [13]. Another project followed the PA program to address the ASM problem in attack helicopters is the Rotorcraft Pilots Associate (RPA) program. The goal of US Army funded RPA program was to develop and demonstrate 
in flight an advanced, intelligent associate system in a nextgeneration attack/scout helicopter [14].

The PA and RPA projects paved the way for other projects to develop and automate the asset management process, namely MAGIC developed by a joint venture of several European universities and companies [15], ISHM developed by NASA for space applications [16], AEGIS, developed by the Honeywell led Abnormal Situation Management (ASM) Consortium in the United States [17], and PAWS developed by a joint venture of Atlantic Canadian universities and National Research Council of Canada (NRC) for oil and gas applications [1], [18], [2], [19], [20]. The most important project among the first five is AEGIS, which proposes a comprehensive asset management framework from an industrial view point. AEGIS built on the experience of military aviation research projects, especially the Pilots Associate (PA) and the Rotorcraft Pilots Associate (RPA) [21]. It is really worth considering the project and its current status, since it is supported by major oil and gas companies (i.e., Shell, Exxon, Chevron, BP, and Nova Chemicals) allied with Honeywell and other automation industry key leaders. Furthermore, it is considered a research imperative to learn from it, in terms of experience, stages being successfully accomplished, limitations, and failures incurred during the course of the project.

The research program life span started from 1994 and will end in 2008, where the program was funded by the National Institute of Standards and Technology (NIST). The program focused on the development of a proof of concept system called AEGIS (Abnormal Event Guidance and Information System). The program successfully demonstrated the feasibility of collaborative decision support technologies in the lab test environment, with a high fidelity simulation model of an industrial manufacturing plant. As far as industrial environment testing is concerned, the focus was on abnormality diagnosis and early warning, and assessing and learning from experience, which resulted in effective operations practices and supporting services.

The AEGIS research program team has achieved several goals and developed a well established abnormal situation management awareness and culture through massive consultation, research, and collaboration with oil and gas industry key leaders. Achievements can be summarized in the following points as presented by the director of advanced development at Honeywell, Mr. A. Ogden-Swift, during the 2005 advanced process control applications for industry workshop (APC 2005) [22]:

- Significant user interface (UI) improvements

- $35 \%$ Reduction in alarm flooding by introducing a new alarm reconfiguration philosophy

- Integration of operation procedures

- Equipment monitoring through intelligent sensor integration

- Fuzzy/PCA early error detection

- Improved operator training

Such achievements were deployed in the new generation of Honeywell's Experion distributed control system. Al- though the 12 year old ASM research program has resulted in a well defined ASM problem in terms of best practices, goals, and limitations, it did not address the following points, which aim to minimize the workload on process operators:

- Full automation of massive process data interpretation

- Full automation of process fault diagnosis and accommodation

- Incorporation of state of the art fault diagnosis techniques which were developed during the past 25 years of academic research

- Reduced manual system configuration by process operators (for example, the operator has to choose the appropriate dataset for process model identification)

- Intelligent techniques such as expert systems to assist operators in the decision making process

Only one technique was used for early fault detection, a statistical technique based on principle component analysis (PCA). To enable this, the operator has to manually adapt for operating point change by choosing the appropriate dataset. In the recent Model Identification Workshop held in the University of New Brunswick [19], Dr. W. Larimore suggested that a better and more comprehensive statistical approach could be used, which is consistent with UNB's approach to PAWS [1], [2], and realized in practice [18].

Having shown the current status of asset management research in both academia and industry, we conclude that the AEGIS ASM research program focused on the bookkeeping and human machine interaction tasks rather than a fully automated and functional asset management holistic approach. The UNB's PAWS research program came to benefit from the success and limitations of AEGIS, to build on its experience, to complement its developed tasks, and to push the envelop by evaluating and incorporating state of the art of fault diagnosis, artificial intelligence (AI) and wireless sensor networks techniques. This will be embedded in a fully automated system architecture, which will better support process operators and improve operability.

\section{THE PETROLEUM APPLICATION OF WIRELESS SYSTEMS (PAWS) PROJECT}

Driven by the technical demand of the offshore oil and gas industry in Atlantic Canada, a joint venture between several Atlantic Canadian universities, the National Research Council of Canada, and local and national companies was established in order to advance wireless systems technology in the oil and gas industries and to assess the feasibility of an intelligent control and asset management system built on a wireless sensor network. The petroleum applications of wireless systems (PAWS) project scope is to develop a control and information management system which consists of two subsystems. The first subsystem is a wireless sensor network which will alleviate the need for data cables in offshore oil rigs and improve flexibility for adding and reconfiguring sensors. The second subsystem intelligently manages the massive data flow from oil rigs and interprets it so as to help operators take more appropriate 


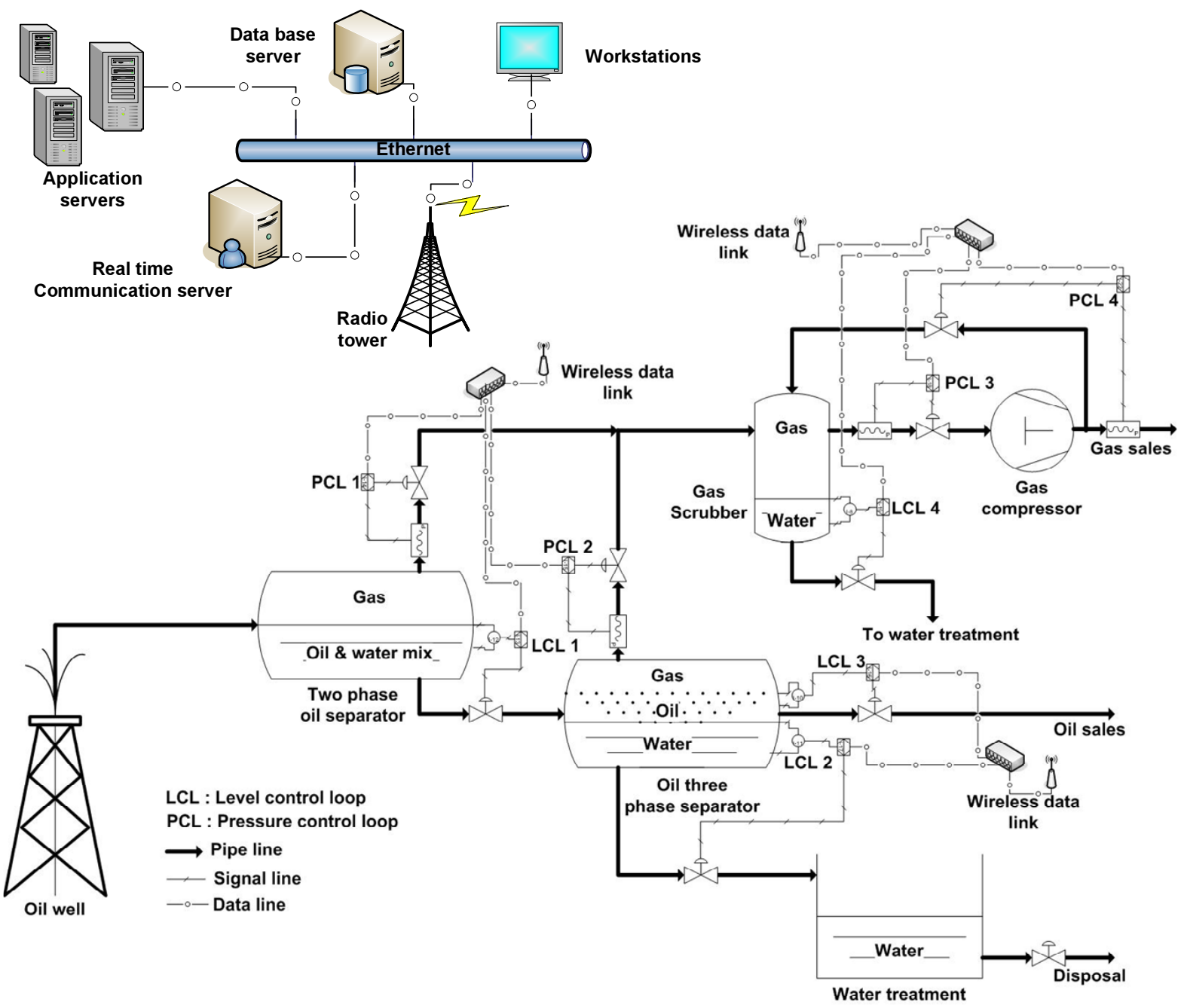

Fig. 1. PAWS Project schematic diagram

decisions during abnormal events and, through intelligent control, improve process economics. As part of the PAWS project, our team is developing an intelligent control and asset management system (ICAM system) to manage the massive information flow from offshore oil rigs. The final developed system will be deployed and validated on a pilot plant which emulates an offshore oil production facility, as illustrated in figure 1.

The pilot plant basically consists of three processes. The first is a two phase separator in which hydrocarbon fluids from oil wells are separated into two-phases to remove as much light hydrocarbon gases as possible. The produced liquid is then pumped to a three-phase separator, where water and solids are separated from oil. Oil is heated in this process to remove as many suspended water droplets from the oil phase as possible. The produced oil is pumped out and sold to refineries and petrochemical plants if it meets the required specifications. Flashed light and medium gases from the separation processes are sent to a gas scrubber where medium hydrocarbon and other liquid remnants are separated from gas and sent back for further treatment.
Produced gas is then compressed and pumped out for sales.

Figure 1 shows the different level and pressure control loops, which maintain the produced oil at the required specifications. As the PAWS project scope suggests, all the process control instrumentations will be hooked up to a wireless communication system. Measured data is transmitted to the control room where the ICAM system will interpret these data for better process control and management. ICAM is composed of a group of servers and operator work stations linked to each other through a high speed ethernet network. The wireless sensor network will be managed by a real time communication server. The database server will store received data in its database after being preprocessed. A group of application servers will be the backbone of ICAM system. The application servers will run the tasks of fault diagnosis, data preprocessing, model identification, fault mitigation and accommodation, human machine interaction, and supervisory control. Each server is a computer cluster, which is a group of loosely coupled computers that work together closely to achieve higher performance, availability, and load balance. This will result 


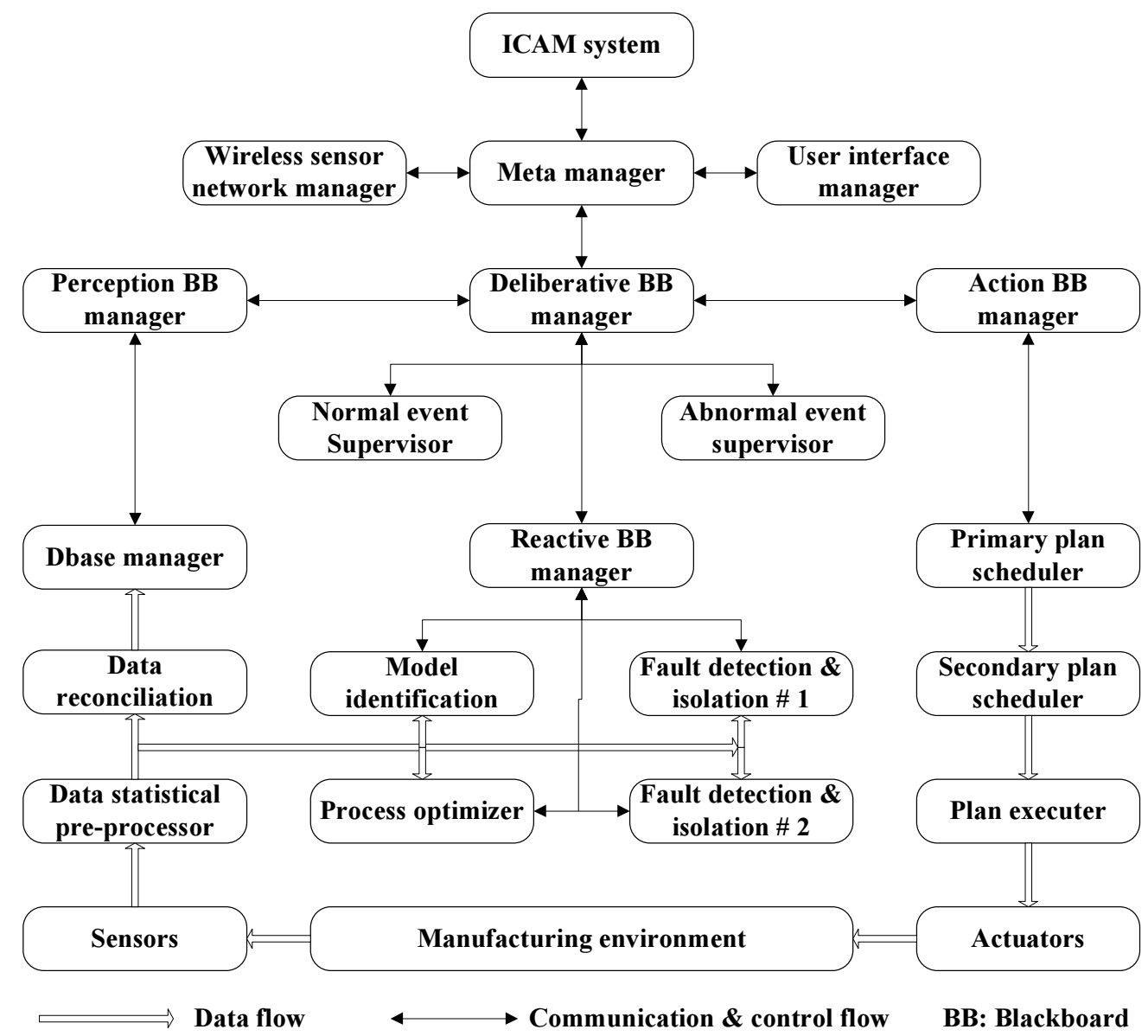

Fig. 2. ICAM system behavioral model

in better internal coordination among the different ICAM servers. ICAM system coordination and internal behavior is discussed in details in the next section.

\section{BEHAVIORAL MODEL OF ICAM SYSTEM}

As illustrated by figure 2, the proposed behavioral model of the ICAM system was built upon our previous work in which we defined the architecture of the system, its functional modules, and its coordination mechanisms, [1], [2]. We adopted Sloman's H-Cogaff architectural scheme because it met most of our system requirements [23]. The behavioral model was drawn as a page hierarchy to make it compatible with hierarchical colored petri net (HCPN) terminology, which will be used to analyze the logical correctness and the dynamic behavior of the system. We follow the top-down approach to explain the behavioral model of ICAM system.

The prime page in the model is called ICAM which contains all the subpages of the system. Each subpage represents an independent agent which interacts with others by means of communications (represented by thin bidirectional arrows). Other agents may process data received from the plant directly (data flow is represented by white thick unidirectional arrows). The meta manager is the main coordinator of the whole system, which guarantees more robust and coherent performance. The meta manager is basically a colored petri net (CPN), which codifies all possible system behaviors and agent interactions as a behavior hierarchy in its full occurrence graph (refer to the next section for its definition). This design decision was taken based upon Durfee's informal theory, which integrates organizational behavior, long term plans, and short term schedules into one coordination framework [24]. Agent behavior is represented in the behavior hierarchy by a single structure, which will use the same message structure communicated between agents. This will result in a better CPN performance instead of having many types of colored tokens (i.e., message structures). Table I illustrates the unified behavior structure.

\begin{tabular}{|c|c|}
\hline Field name & Field content \\
\hline Tag & Message ID \\
From & Sender \\
To & Recipient \\
What & Goals \\
How & Plans \\
When & Schedule \\
How long & Task length \\
Why & Meta reasoning \\
\hline
\end{tabular}

TABLE I

STRUCTURE OF BEHAVIORAL MESSAGE 
The meta manager interacts with other ICAM agents through a group of distributed black board agents, which act as a post office. Each blackboard (BB) agent consists of a global data repository and a control mechanism, which processes the different received messages and notifies other agent about newly available messages. Once the meta manager receives a message from any of the $\mathrm{BB}$ agents, It monitors the logical behavior by comparing the sender and the recipient identities and status with its current marking state. Furthermore it assesses the dynamic behavior of the system in this event by assigning a time out for the recipient to respond. Should the recipient not respond within the preset time out, the meta manager proposes an alternative internal action. Each BB agent broadcasts messages to the recipients and to the higher level $\mathrm{BB}$ agents. The highest level $\mathrm{BB}$ agent would send the massage to the meta manager for further processing. It is worth mentioning that the detailed description of the meta manager behavior will become more clear as the behavioral model development of ICAM system gets closer to meeting the required specifications.

The deliberative BB manager interacts with the lower level BB managers and the two case based reasoning (CBR) supervisory agents of ICAM. The main CBR supervisor manages the system during normal manufacturing operations. When a certain product specification is required, the main CBR supervisor retrieves a set of cases that best match the required quality specs. If the matching process is successful, the plan is sent to the deliberative $\mathrm{BB}$ manager which in turn communicates it to action $\mathrm{BB}$ manager for execution. If not, the closest matching case is chosen and adapted by using model-based optimization, in which the main CBR supervisor collaborates with the model identification and optimization agents to generate the optimal recipe and operating conditions (e.g., pressure and temperature). Such collaboration process is achieved through the deliberative and reactive BB managers. The plan is sent to the user interface agent for further modifications by process operators if needed. Once the plan has been approved it is then sent to the action $\mathrm{BB}$ manager for execution. The meta manager is acknowledged in every step, to guarantee a coherent internal coordination.

The second CBR supervisor is a backup agent to manage the system in case of faulty operations. When a fault happens (e.g., a sensor or actuator failure), the backup agent receives fault assessments from the different fault detection and isolation (FDI) agents through the reactive $\mathrm{BB}$ manager. Based on such assessments, the supervisor retrieves the most closely matching case from its library. Consequently, it alarms the user interface through the appropriate BB managers about the fault, its possible causes, and recommended mitigating actions for operator feedback and approval. The backup CBR supervisor may interfere directly in critical situations in collaboration with the main supervisor and the meta manager. This would prevent the system performance from deteriorating excessively and would keep it in an acceptable state.

Four reactive agents respond directly to events as they are continuously updated with new data about the external environment. A model identification agent estimates new process parameters to improve the system knowledge about the process, should it receive a message from the perception BB manager to do so. The production efficiency is improved by a process optimization agent, monitored by the main CBR supervisor as discussed earlier. When abnormal events occur, two FDI agents collaborate with the backup supervisor, and other BB managers to detect and isolate such faults. The first agent to detect abnormal process variations is the statistical data monitor, which alerts FDI and backup agents through appropriate BB managers. Again the meta manager is acknowledged at every step to ensure coherent coordination among the agents. One of the FDI agents exploits the generalized parity space (GPS) to generate a set of directional residuals, from which process faults can be determined [25], [18], [26]. The other FDI agent is based on a neuro-fuzzy modeling and symptom generation approach [27].

Data from the external plant are received by the statistical data monitoring agent, which preprocesses the data by removing undesired discrepancies. Data is then reconciled with material balance laws in the data reconciliation agent [28], [29]. The data base manager then stores processed data in its database. The perception BB manager connects these data processing agents with the system. Plans received by the action BB manager are sent to the main and secondary scheduling agents where plans are decomposed into tasks with shorter time frames and in accord with the sub-processes of the plant. Subtasks are then executed by the execution agent. Massive data flow is handled by a wireless sensor network agent, which manages real time communications between the control room and the offshore oil facility. Process-critical information is pro-actively presented to process operators in a timely manner through the user interface agent.

\section{ICAM SYSTEM DEVELOPMENT PLAN}

Having discussed the behavioral model of the ICAM system, it is very crucial to prepare an implementation plan and choose the appropriate development tools. The implementation plan is a preliminary one for prototyping and performance analysis purposes. Industry consultation will determine the final system requirements and deployment plan. Our preliminary implementation plan is composed of the following phases:

1) ICAM system logical behavior analysis using the colored petri nets (CPN) approach: The CPN modeling approach combines Petri nets and programming languages. Petri nets provide the foundation of the graphical notation and the semantical foundation for modeling concurrency, synchronization, and communication in systems. CP-nets have a module concept allowing CPN models to be organized into several modules (called pages). The module concept is hierarchical, allowing a module to have a number of submodules and allowing a set of modules to be composed to form 
new modules, as illustrated by figure 2 for the ICAM system page hierarchy. CPN tools is a development environment for constructing and analyzing CP nets. It supports state space (reachability) analysis of CPN models. The basic idea in state spaces is to calculate all reachable states and state changes of the system and represent these as a directed graph called an occurrence graph. The state space of a CPN model can be used to verify a number of dynamic properties of the system under consideration such as liveness, boundedness, and fairness [30].

2) ICAM system deployment scheme using the message passing interface (MPI) parallel programming model: The message passing model is a parallel programming approach which posits a set of processes that have only local memory but are able to communicate with other processes by sending and receiving messages. The MPI model has many advantages such as expressivity, ease of debugging, and most importantly high performance [31]. MPI is a specification and a library which provides the infrastructure for communications among several parallel computational processes. MPI gives system designers the freedom to implement their own protocols that best fit their systems' requirements. MPI can be used on a PC cluster or a network of workstations, which paves the way for ICAM system rapid prototyping and deployment. The Cornell Multitask Toolbox for MATLAB (CMTM), which was developed by Cornell Theory Center at Cornell University, will be used for ICAM fast prototyping and development.

3) Canonical variate analysis (CVA) integration for massive data flow processing: Massive dataflow from industrial process should be preprocessed so as to remove any inconsistencies, to model the process, and to generate better process measurements in accordance with mass balance laws. The canonical variate analysis (CVA) approach is a well established statistical approach, which can meet the dataflow preprocessing requirements mentioned earlier. This decision was made as a result of a three day model identification workshop held at the University of New Brunswick. Dr. W. Larimore suggested the integration of the CVA-based Adaptx package with ICAM would result in robust data preprocessing and modeling compared with other approaches. The Adaptx package was developed during the past 25 years of research in the area of statistical data analysis.

4) Bottom-up ICAM system rapid prototyping using the MATLAB simulation environment:

In order to have the ICAM system requirements deployed in a real-world system, a prototype has to be developed. We will follow the bottom-up development approach in which a simple prototype will be developed. Once the simple prototype performance and logical behavior have been verified and validated, new agents can be added to the system. Figure 3 illustrates

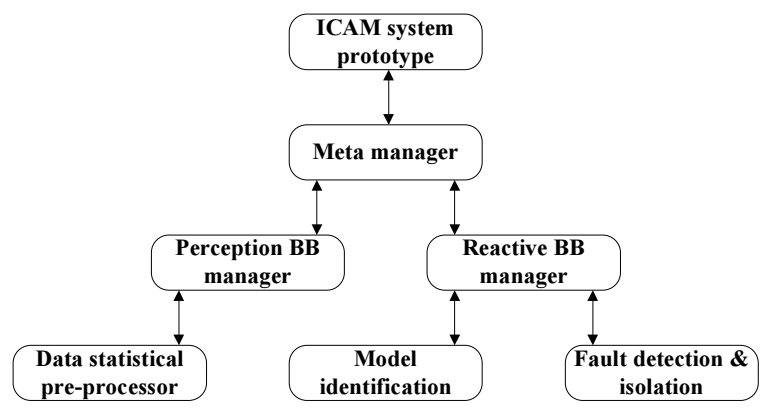

Fig. 3. CPN hierarchy page of ICAM system prototype

the CPN hierarchy page of the simplified ICAM prototype. When the data statistical preprocessor detects a change in the operating point or an abnormal change in data, it alerts the perception BB manager which in turn sends appropriate messages to the meta manager and the reactive $\mathrm{BB}$ manager. Based upon the recipient ID of the different messages, the reactive $\mathrm{BB}$ manager sends messages to the model ID and FDI agents to further identify the nature of the data change. If the change is in the process operating point, the FDI agent asks the model ID agent to update the process model parameters. If the change is a process fault (i.e., a sensor or actuator fault), the FDI agent detects the nature of the fault and acknowledges the reactive $\mathrm{BB}$ manager for further processing. For every event that occurs, the meta manager is acknowledged, which in turn monitors the logical behavior of the system by ensuring that every sequence of events is a branch of occurrence graph of the meta manager CPN. The communicated messages have a unified format, which makes that system CPN performance analysis easier and smoother.

The different blackboard BB managers of the system have the same scheme, which will be designed based on the MPI framework. The BB manager logical structure and dynamic performance will be analyzed in the CPN tool analysis environment. The ICAM prototype will be deployed as distributed MATLAB computational modules, which will run on an network of several Windows XP workstations. MATLAB sessions will exchange messages by using an off-theshelf MPI MATLAB toolbox. The different computational agents will employ different approaches based on the agent's task. The model ID agent will use the canonical variate analysis CVA based subspace state space approach, whereas the FDI agent will be based on the generalized parity vector (GPV) concept. Finally the statistical data preprocessor will employ the principle components analysis approach. The system will be interfaced with an oil production pilot plant offered by the College of North Atlantic (CNA), where the ICAM system performance will be verified and validated.

5) College of North Atlantic (CNA) oil production pilot 


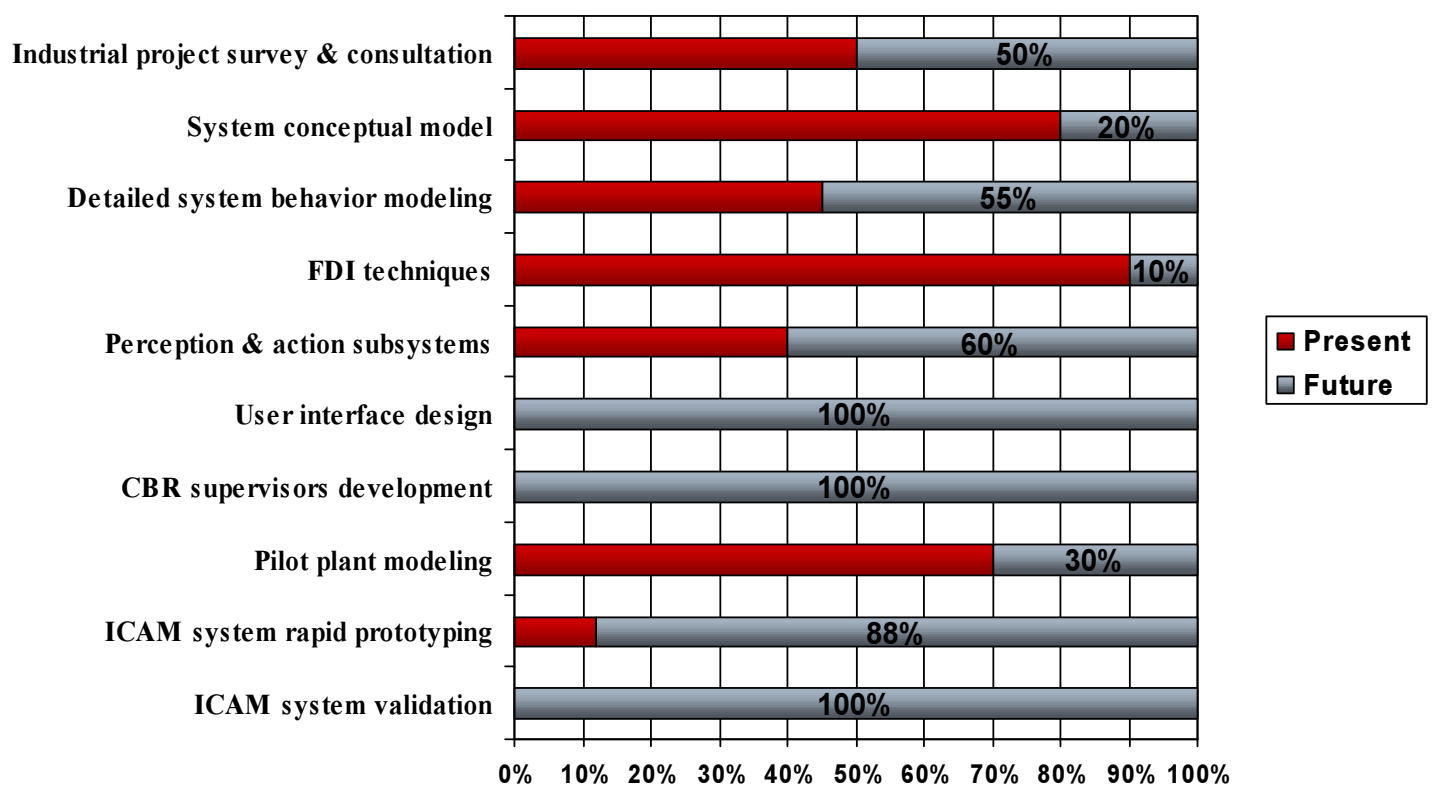

Fig. 4. ICAM system development progress status

plant development: As far as the pilot plant, we are suggesting upgrading its equipment and instrumentations, especially the sensors. Furthermore, we will consult with our industrial partners, mainly $\mathrm{ABB}$, to design a standard mini SCADA system for the CNA pilot plant to meet industrial standards and future wireless upgrade option. In addition to installing a LAN of workstations, we will use Matlab simulation package including the OPC toolbox, which will act as an interface between the workstations and the pilot plant mini SCADA system. This would be very productive and would guarantee good development and utilization process of the CNA facility even after PAWS is completed.

\section{PROJECT STATUS AND FUTURE WORK}

As part of the PAWS joint venture, and as the leader in developing the ICAM system, we have formed a task force of five graduate students at the University of New Brunswick to address the integration of control and asset management for a large process industry application. As illustrated in figure 4, the ICAM development process was broken into different tasks. Starting in January 2004, the project has progressed well. A rigorous review of the available system architectures and their characteristics has been done so as to match them with proposed system requirements. Consultation with industrial and automation partner companies is in progress to produce final specifications and documentation for the architectural level and execution platform in order to meet industry standards. The system conceptual model was chosen and has an almost complete description [1], [2].

Three team members were assigned the task of developing, testing and evaluating the different proposed FDI techniques. The FDI agents development task has successfully met several major goals, such as quick detection and isolation, isolability, robustness and disturbance decoupling. The task of evaluating the different data processing techniques which will be incorporated in the perception subsystem is assigned to another of the team members, who is presently focussing on data preconditioning and reconciliation. The task of modeling the pilot plant, which will be used to validate the system performance, is in the final modeling stage. Three day model identification was held at UNB, where Dr. W. Larimore gave a massive survey of statistical data processing and modeling approaches, and introduced his CVA-based Adaptx package as the state of the art of data processing tool. We have initiated the rapid prototyping of ICAM system as discussed in this paper. We are planning to design the plan schedulers based on the receding horizon control approach.

A three day PAWS workshop at Cape Breton University (CBU) was held in August 2005, where the project partners including UNB, CBU, CNA, and NRC met and discussed the project progress and future plans. The workshop addressed the wireless sensor network development task with several Canadian university representatives in the area of wireless sensor communications.

When compared with the AEGIS/ASM research project, it is obvious that the ICAM/PAWS research project complements and builds on the experiences of the AEGIS/ASM project. It is not intended to design a new industrial control system technology from scratch. However, the PAWS project objective is to build on present-day industrial distributed control systems and utilize the current industrial standards, which has been developed and matured in the past 25 years. We believe that the successful design and development of the proposed system will lay a cornerstone in the area of complex intelligent system development, and will open the doors for other applications such as distributed 
power plant management.

\section{ACKNOWLEDGEMENT}

This project is supported by Atlantic Canada Opportunities Agency (ACOA) under the Atlantic Innovation Fund (AIF) program. The authors gratefully acknowledge that support and the collaboration of Cape Breton University (CBU), the National Research Council (NRC) of Canada, and the College of the North Atlantic (CNA). The authors also acknowledge the support of the Natural Sciences and Engineering Research Council of Canada (NSERC) for funding the first author's research. We also would like to extend our thanks to Dr. Wallace Larimore for his support and invaluable suggestions.

\section{REFERENCES}

[1] J. H. Taylor and A. F. Sayda, "Intelligent information, monitoring, and control technology for industrial process applications," in The 15th International Conference on Flexible Automation and Intelligent Manufacturing (FAIM), Bilbao, Spain, July 2005.

[2] — , "An intelligent architecture for integrated control and asset management for industrial processes," in Proc. IEEE International Symposium on Intelligent Control (ISIC05), Limassol, Cyprus, June 2005.

[3] V. Venkatasubramanian, R. Rengaswamy, S. N. Kavuri, and K. Yin, "A review of process fault detection and diagnosis part 1,2,3," Computer \& Chemical Engineering, vol. 27, no. 3, pp. 293-346, 2003.

[4] R. J. Patton, "Fault-tolerant control systems: The 1997 situation," in IFAC Symposium on Fault Detection Supervision and Safety for Technical Processes, R. J. Patton and J. Chen, Eds., vol. 3. Kingston Upon Hull, UK: IFAC, August 1997, pp. 1033-1054.

[5] P. M. Frank and B. Köppen-Seliger, "New developments using AI in fault diagnosis," Engineering Applications of Artificial Intelligence, vol. 10, no. 1, pp. 3-14, 1997.

[6] R. L. Moore and M. Kramer, "Expert systems in online process control," in Proceedings of the 3rd international conference on chemical process control, Asilomar, California, 1986.

[7] J. H. Taylor, L. P. Harris, P. K. Houpt, H.-P. Wang, and E. S. Russell, "Intelligent processing of materials: Control of induction-coupled plasma deposition," in Advanced Sensing, Modelling, and Control of Materials Processing. Warrendale, PA: Ed. by E. F. Matthys and B. Kushner, TMS Publications, 1991.

[8] A. Newell, Unified theories of cognition. Cambridge, MA: Harvard University Press, 1990.

[9] F. E. Ritter, N. R. Shadbolt, D. Elliman, R. Young, F. Gobet, and G. D. Baxter, "Techniques for modeling human performance in synthetic environ-ments: A supplementary review," Human Systems Information Analysis Center (HSIAC), formerly known as the Crew System Ergonomics Information Analysis Center (CSERIAC), Wright-Patterson Air Force Base, OH, Tech. Rep., 2003.

[10] E. Durfee and T. Montgomery, "MICE: A flexible test bed for intelligent coordination experiments," in Proceedings of the 9th workshop on distributed AI, Rosario, Washington, September 1989.

[11] M. J. Wooldridge, An introduction to multiagent systems. Chichester, England: Wiley, 2002.

[12] R. L. Small and C. W. Howard, "A real-time approach to information management in a pilot's associate," in Proceedings of Digital Avionics Systems Conference. IEEE/AIAA, 14-17 Oct 1991, pp. 440-445.

[13] S. B. Banks and C. S. Lizza, "Pilot's associate: a cooperative, knowledge-based system application," IEEE Expert, vol. 6, no. 3, pp. 18-29, June 1991.

[14] C. A. Miller and M. D. Hannen, "Rotorcraft pilot's associate: Design and evaluation of an intelligent user interface for cockpit information management," Knowledge-Based Systems, vol. 12, no. 8, pp. 443456, Dec 1999.

[15] B. Köppen-Seliger, T. Marcu, M. Capobianco, S. Gentil, M. Albert, and S. Latzel, "MAGIC: An integrated approach for diagnostic data management and operator support," in Proceedings of the 5th IFAC Symposium Fault Detection, Supervision and Safety of Technical Processes - SAFEPROCESSO5, Washington D.C., 2003.
[16] J. Schmalzel, F. Figueroa, J. Morris, S. Mandayam, and R. Polikar, "An architecture for intelligent systems based on smart sensors," IEEE Transactions on Instrumentation and Measurement, vol. 54, no. 4, pp. 1612-1616, August 2005.

[17] T. Cochran, P. Bullemer, and I. Nimmo, "Managing abnormal situations in the process industries parts 1, 2, 3," in NIST Proceedings of the Motor Vehicle Manufacturing Technology (MVMT) Workshop, Ann Arbor, MI, 1997.

[18] M. Omana and J. H. Taylor, "Robust fault detection and isolation using a parity equation implementation of directional residuals," in IEEE Advanced Process Control Applications for Industry Workshop (APC2005), Vancouver, Canada, May 2005.

[19] W. Larimore, in Multivariable System Identification Workshop. Fredericton, New Brunswick: University of New Brunswick, 31 October - 2 November 2005.

[20] C. Smith, C. Gauthier, and J. H. Taylor, in Petroluem Applications of Wireless Sensors (PAWS) Workshop. Sydney, Nova Scotia: Cape Breton University, 22-23 August 2005.

[21] E. L. Cochran, C. Miller, and P. Bullemer, "Abnormal situation management in petrochemical plants: can a pilot's associate crack crude," in Proceedings of the 1996 IEEE National Aerospace and Electronics Conference, NAECON, vol. v2. Dayton, KY, USA: IEEE, Piscataway, NJ, USA, May 20-23 1996, pp. 806-813.

[22] A. Ogden-Swift, "Reducing the costs of abnormal situations ... the next profit opportunity," in IEEE Advanced Process Control Applications for Industry Workshop (APC2005), Vancouver, Canada, May 2005.

[23] A. Sloman, "Varieties of affect and the CogAFF architecture schema," in proceedings of symposium on Emotions, Cognition, and Affective Computing at the AISB'01 convention, York, UK, 2001.

[24] E. Durfee and T. Montgomery, "Coordination as distributed search in a hierarchical behavior space," IEEE Transactions on Systems, Man, and Cybernetics, vol. 21, no. 6, pp. 1363-1378, 1991.

[25] N. Viswanadham, J. H. Taylor, and E. C. Luce, "A frequency domain approach to failure detection and isolation with application to GE21 turbine engine control system," Control Theory and Advanced Technology, vol. 3, no. 1, pp. 45-72, 1987.

[26] M. Omana and J. H. Taylor, "Enhanced sensor/actuator resolution and robustness analysis for fdi using the extended generalized parity vector technique," in accepted for the American Control Conference. Minneapolis, Minn.: IEEE, 14-16 June 2006.

[27] J. Hi, "Neuro-fuzzy based fault diagnosis for nonlinear processes," Master's thesis, University of New Brunswick, May 2006.

[28] J. H. Taylor and M. Laylabadi, "A novel adaptive nonlinear dynamic data reconciliation and gross error detection method," in accepted for the IEEE Conference on Control Applications. Munich, Germany: IEEE, October 4-6 2006.

[29] M. Laylabadi and J. H. Taylor, "Anddr with novel gross error detection and smart tracking system," in 12th IFAC Symposium on Information Control Problems in Manufacturing. Saint-Etienne, France: IFAC, May 17-19 2006.

[30] L. M. Kristensen, J. B. Jrgensen, and K. Jensen, Lectures on concurrency and petri nets: advances in petri nets. Springer-Verlag, 2004, ch. Application of coloured petri nets in system development, pp. 626-685.

[31] W. Gropp, E. Lusk, and A. Skjellum, Using MPI: portable parallel programming with the message-passing interface, 2nd ed., ser. Scientific and Engineering Computation, J. Kowalik, Ed. Cambridge, Massachusetts: MIT Press, 1999. 